\title{
Borderline Intermediate Thickness Cutaneous Melanoma Class B: Isn't it Time for Personalised One Step Surgical Approach as Standard Clinical Behaviour?
}

\author{
Kandathil LJ ${ }^{1}$, Oliveira $\mathbf{N}^{1}$, Patterson $\mathrm{JW}^{2}$, Tchernev $\mathbf{G}^{{ }^{*}}$ \\ ${ }^{1}$ Medical Student, Faculty of Medicine, Medical University of Sofia, Bulgaria \\ ${ }^{2}$ University of Virginia, USA
}

${ }^{3}$ Onkoderma-Policlinic for Dermatology, Venereology and Dermatologic Surgery, Sofia, Bulgaria

Received: June 10, 2021; Accepted: June 16, 2021; Published: June 19, 20211

*Corresponding author: Georgi Tchernev, Onkoderma-Clinic for Dermatology, Venereology and Dermatologic Surgery, General Skobelev 26 , E-mail: georgi_tchernev@yahoo.de

\section{Case Report}

We present a 40 -year-old female who visited our clinic with a solitary lesion on the posterolateral aspect of the lower left leg (Figure 1a). She noticed a progressive change in the size and shape of the lesion and decided to consult a dermatologist in March 2021. The patient had a history of Hashimoto's thyroiditis that was well-controlled onlevothyroxine. No other comorbidities were reported and she was otherwise healthy. During the clinical examination a single pigmented patch measuring 2.5 $\mathrm{cm}$ in greatest diameter was identified. Morphologically the lesion was asymmetrical with irregular borders and uneven colour. At the centre, an exudative, ulcerated nodule was also noted (Figure1a-f). Clinical and dermatoscopic findings were consistent with the diagnosis of a superficial spreading cutaneous melanoma. Ultrasound diagnostics of the abdominal cavity and retroperitoneal organs showed no signs of tumor spread. Chest radiography was also within normal limits. Laboratory testing showed an elevated uric acid level of $456 \mu \mathrm{mol} / \mathrm{l}$ (reference range $142-340 \mu \mathrm{mol} / \mathrm{l}$ ), but otherwise all other parameters were normal. Following the recommended American Joint Committee on Cancer (AJCC) guidelines, we performed a primary resection with $0.5 \mathrm{~cm}$ margins in all directions. The resected tissue was subsequently sent for histopathological evaluation and confirmed the diagnosis of borderline intermediate thickness malignant melanoma - class B, 4 mm Breslow thickness, Clark IV, (pT4BN0M0) (Figure2a-d). There was high mitotic activity but no spontaneous regression, insignificant lymphocytic stromal reaction and clear resection margins. Post diagnostic workup, including chest and abdominal CT, showed no signs of metastatic dissemination. One week later, the patient was sent to the National Oncology Hospital for re-excision and Sentinel Lymph Node Biopsy (SLNB). The re-excision of additional $2 \mathrm{~cm}$ from the previous surgical scar was conducted in parallel with removal of the draining sentinel lymph node (Figures $3 \mathrm{a} \& 3 \mathrm{~d}$ ). The closure of the defect after re-excision led to the unfortunate complication of wound dehiscence and failure to close successfully (Figure $3 \mathrm{~b}-\mathrm{c}$ ). However, after several sessions of debridement, cleansing, rebandaging and administration of antibiotics, there was visible improvement with subsequent resolution(Figure $3 d-f$ ). Since that time, she has been in excellent condition, and no complications have been reported to date.

The guidelines set by the AJCC and the European Consensus define intermediate thickness melanomas as 1-4 mm in depth and recommend particular two-step treatment strategies (Table 1) $[1,5]$. The American guidelines suggest treating all intermediatethickness cutaneous melanomas within this $1-4 \mathrm{~mm}$ thickness range with a wide variable margin of $1-2 \mathrm{~cm}$, but always with the parallel performance of Sentinel Lymph Node Biopsy (SNLB)in two surgical sessions[1].

For the first time, we introduce two subgroups of medium-thick skin melanomas:(1) intermediate-thickness class A melanomas, 1 to $2 \mathrm{~mm}$ in tumor thickness, and (2) class B melanomas, between 2 and $4 \mathrm{~mm}$ in tumor thickness. According to European guidelines, the total resection field for class A lesions is $1 \mathrm{~cm}$ (achieved in two surgical sessions), with removal of a draining lymph node being highly recommended during the second surgical session. For class B lesions, the approach is similar, but the total resection field (again within two surgical sessions) should be $2 \mathrm{~cm}$, the second surgical session being combined with parallel removal of a draining lymph node. The main problem with the AJCC guidelines stems from the recommendations on the resection field for melanomas with a thickness between 1 and 2 $\mathrm{mm}$; namely, that it can vary between 1 and $2 \mathrm{~cm}$ [1]. In practice, the AJCC recommendations for surgical treatment of melanomas with a thickness of $1.00 \mathrm{~mm}$ and those with a thickness of 4.00 $\mathrm{mm}$ are identical [1]. The differences inmanagement of these types of lesionsconsist only of the recommended (but not 

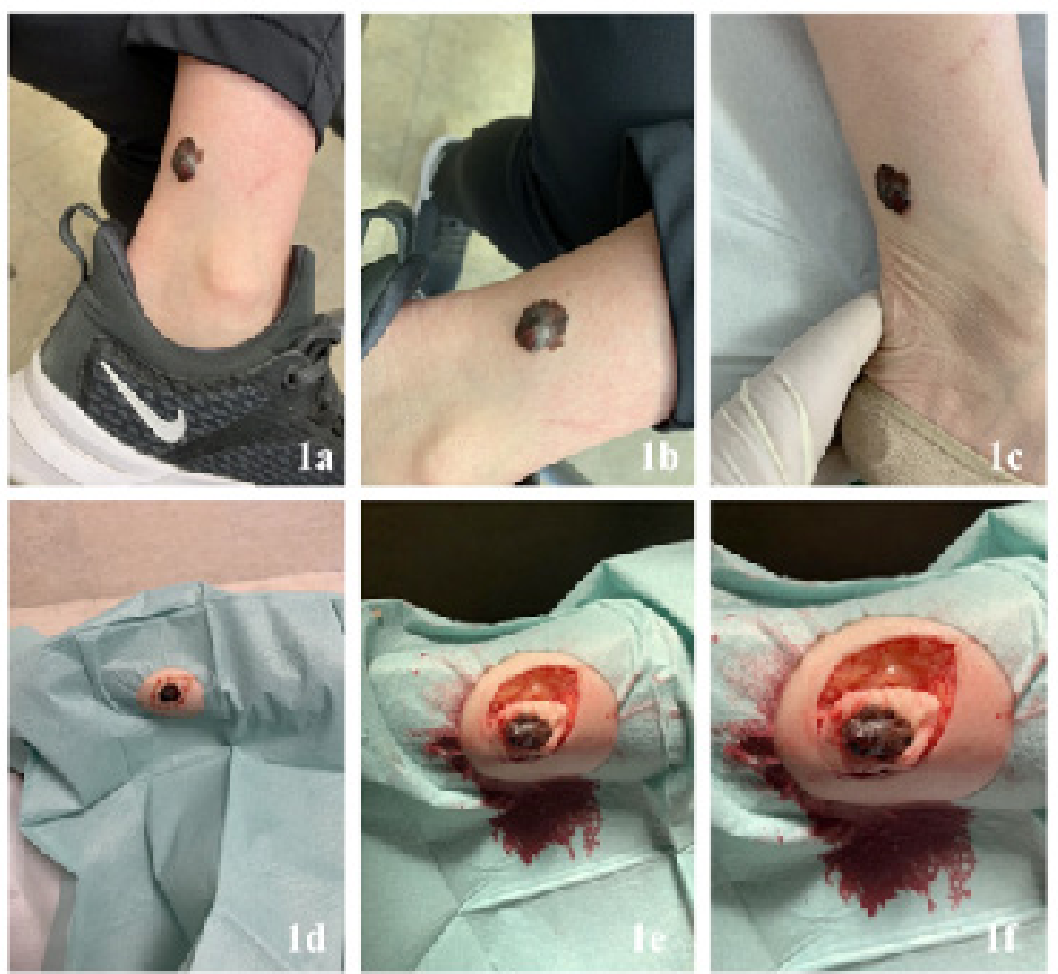

Fig 1a-c: Asymmetric, irregular, hyperpigmented lesion of $2.5 \mathrm{~cm}$ in diameter with an ulcerated nodular appearance located on the posterolateral lower left leg.

Fig1d: Preoperative preparation and marking with additional $0.5 \mathrm{~cm}$ surgical margin around the entire lesion.

Fig1e-f: Intraoperative appearance of the primary incision.

mandatory)removal of the draining lymph node for melanomas between 1 and $2 \mathrm{~mm}$ thickness [1].

The treatment of intermediate thick cutaneous melanomas of Class A and Class B could be considered not completely adequate, and actually it overlaps to a large extent with that of thick melanomas $-2 \mathrm{~cm}$ total resection in two surgical sessions; the determination of a draining lymph node in this lesion is again a matter for discussion [1]. In practice, it turns out that the surgical treatment of thick melanomas and intermediate thickness cutaneous melanomas - Class A and Class B - is very similar. The additional lack of synchronization between clinically measured and histologically established distances from tumor tissue remains an open question [1]. The question could be raised: why do these guidelines exist at all? But analysis of this information suggests another important question: why do equally treated surgical melanomas show different tendencies to metastasize and different prognoses in general? The reason should be sought in the lack of unification of the criteria in the above guidelines. Whether this situationis something that has been desired, deliberately ignored,or is simply accidental remains unclear at this time.

The lack of specificity for the guidance for clinicians when dealing with such a wide range of tumors may lead to much confusion, especially as tumor thickness is such an important indicator of disease progression and prognosis [2-4]. Given such a generalized approach to the treatment of cutaneous malignant melanomas, we are compelled to summarize the potential flaws that the AJCC recommendations fail to address:

1. Patients that fall under the broad category of 1-4 mm intermediate tumor thickness present a wide array of prognostic characteristics and tumor heterogeneity,and yet are all subjected to the same treatment recommendations. (i.e., a patient with tumor thickness of $1.01 \mathrm{~mm}$ may have a treatment strategy identical to that of another patient with tumor thickness of 4.00 mm or even more.

2. Patients with borderline thickness class $A(2 \mathrm{~mm})$ and class B $(4 \mathrm{~mm})$ melanomas are once again treated equally with no distinction in the form of a personalized treatment strategy.

3. The variability of the guidelines allows for errors where a borderline thickness cutaneous melanomas $(1.01 \mathrm{~mm})$ may potentially be treated in the same way as a thick $(>4 \mathrm{~mm})$ melanomas: $2 \mathrm{~cm}$ complete surgical margin plus sentinel LN [1].

The potential implications for such an ambiguous recommendation fall on the clinician, who will more often than not choose to remove the tumor with a greater (and possibly excessive) margin. This could lead to issues such as further surgical complications, additional trauma to the patient, and 
Table I : AJCC recommandations (Swetter et al., 2019)

\begin{tabular}{|c|c|}
\hline Breslow thickness & Recommended surgical margins/ AJCC \\
\hline Melanoma in situ & $0.5 \mathrm{~cm}$ (primary excision with $0.5 \mathrm{~cm}$ in all directions, followed by secondary excision) \\
\hline$<1 \mathrm{~mm}$ & $0.5 \mathrm{~cm}$ primary excision (followed by secondary excision with $0.5 \mathrm{~cm}$ in all directions) \\
\hline $1.01-2.0 \mathrm{~mm}$ & $0.5 \mathrm{~cm}$ primary excision (followed by secondary excision with $0.5-1.5 \mathrm{~cm} /$ with SLNB) \\
\hline $2 \mathrm{~mm}-4 \mathrm{~mm}$ & $0.5 \mathrm{~cm}$ primary excision (followed by secondary with $1.5 \mathrm{~cm}$ in all directions/ with SLNB) \\
\hline$>4 \mathrm{~mm}$ & $0.5 \mathrm{~cm}$ primary excision (followed by secondary excision with $1.5 \mathrm{~cm}$ in all directions/ without SLNB if \\
\hline \begin{tabular}{c} 
nodes not enlarged/matter of discussion) \\
\hline
\end{tabular}
\end{tabular}

Table II: One step Melanoma surgery (OSMS) recommendation (Tchernev et al., 2019)

\begin{tabular}{|c|c|}
\hline Breslow thickness & Recommended surgical margins/ Tchernev et al. \\
\hline Melanoma in situ & $1.0 \mathrm{~cm}$ (clinical/dermatoscopic evaluation obligate/ if possibility for echographical examination-from benefit) \\
\hline$<1 \mathrm{~mm}$ & $1.0 \mathrm{~cm}$ (clinical/dermatoscopic evaluation obligate/ if possibility for echographical examination-from benefit) \\
\hline $1.01-2.0 \mathrm{~mm}$ & $1.0 \mathrm{~cm}$ (with SLNB), (echographic tumor thickness measurement preoperatively) \\
\hline $2 \mathrm{~mm}-4 \mathrm{~mm}$ & $2.0 \mathrm{~cm}$ (with SLNB), (echographic tumor thickness measurement preoperatively) \\
\hline$>4 \mathrm{~mm}$ & $\begin{array}{l}2.0 \mathrm{~cm} \\
0 . \quad \text { If no enlarged lymph nodes- } 2 \mathrm{~cm} \text { resection is sufficient } \\
\text { a. Presence of enlarged lymph nodes- to be removed together with the re excision of primary } \\
\text { tumorous tissue! }\end{array}$ \\
\hline
\end{tabular}

aesthetic consequences, to name a few.

The European Consensus advocates for more precise treatment recommendations for intermediate thickness melanomas but with the same two step approach as the AJCC [5].According to the European evidence-based guidelines, tumors ranging from 1-2mm thickness, considered as class A, are treated with a total of $1 \mathrm{~cm}$ surgical excision together with sentinel lymph node biopsy (SLNB). Class B tumors, with a thickness of $2-4 \mathrm{~mm}$, are treated with a $2 \mathrm{~cm}$ surgical safety margin combined with a SLNB [5].

The personalized one step (OSMS) surgical approach we have previously suggested aims to follow a more precise and tailored treatment plan that reaches the intended result of both recommended guidelines but in one surgical session [4,6].Our proposed guidelines follow the intended European framework of the corresponding surgical safety margins for tumor thickness, alongside a SLNB (Table 2). The OSMS identifies the two borderline intermediate thickness melanomas class $\mathrm{A}$ and $\mathrm{B}$ and suggests a more adequate recommendation for their management in one surgical session (i.e., $1 \mathrm{~cm}$ with SLNB for class A in one session and $2 \mathrm{~cm}$ for class $\mathrm{B}$ in one session) (Table 2).

Our presented patient with a $4 \mathrm{~mm}$ Breslow thickness could be treated with a defined $2 \mathrm{~cm}$ surgical margin in all directions in one session if we were to employ the OSMS regimen. Although histopathological verification of tumor thickness is the gold standard and the primary reason for employing a two-step approach, the use of high-resolution ultrasound to determine preoperative thickness is being increasingly used and has shown to correlate well with histologic Breslow thickness [3,4-
7].In fact, correlation of the complete clinical, dermatological and high-resolution ultrasound data provides a highly accurate preoperative estimation of Breslow thickness $[4,6]$.

The greatest advantage to OSMS is eliminating the need for a second procedure, thus saving the patient time, travel to the specialist center, the need to leave work and, most importantly, cost[4,6-9].With a single surgical session, there is also a twofold reduction in surgical complications, psychological trauma and discomfort for the patient. The patient is also more inclined to comply with a single stage treatment as the requirement of a second surgery often puts doubt on the success of the initial procedure, even if they may be well informed about the entire 2-step process. Especially for lesions that are clinically and dermatoscopically indicative for cutaneous melanomas, personalized or one-step melanoma surgery should be clearly preferred. Preoperative measurement of tumor thickness in these cases is of paramount importance.

Through this clinical case, we propose the use of a personalized one step surgical approach as the best melanoma treatment algorithm. The patient we have reported would fall under the class B category of borderline thickness melanoma and would be treated with a $2 \mathrm{~cm}$ surgical margin and SLNB in one surgicalsession. The patient-centered one step melanoma surgery provides multiple benefits including psychological, physical and financial relief, while meeting the same requirements as the twostep proposal within the European and AJCC guidelines. 

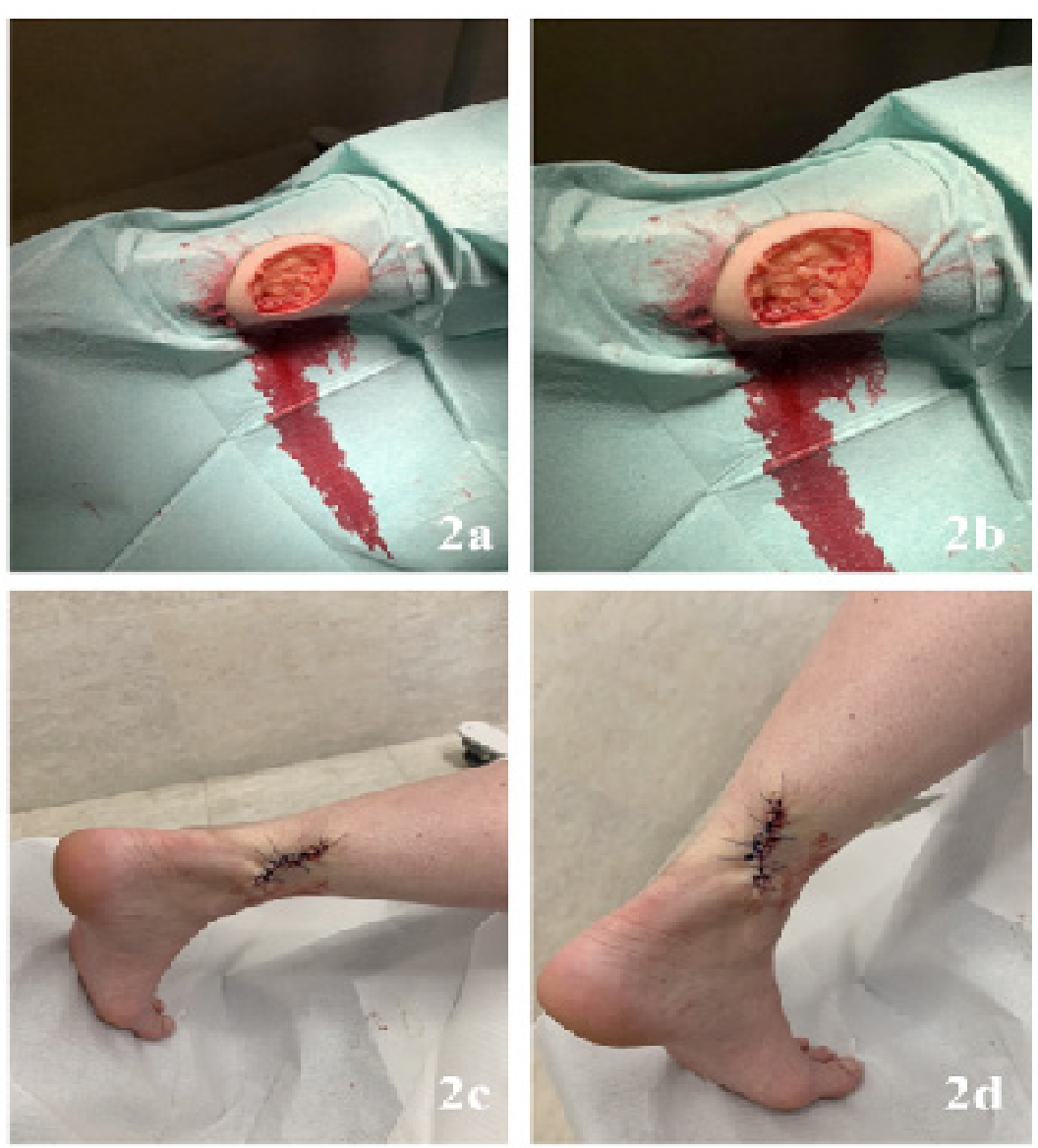

Fig2a-b: Intraoperative view after primary elliptical excision \& preparation of peripheral tissue for closure.

Fig 2c-d: Closure of surgical defect by single interrupted sutures
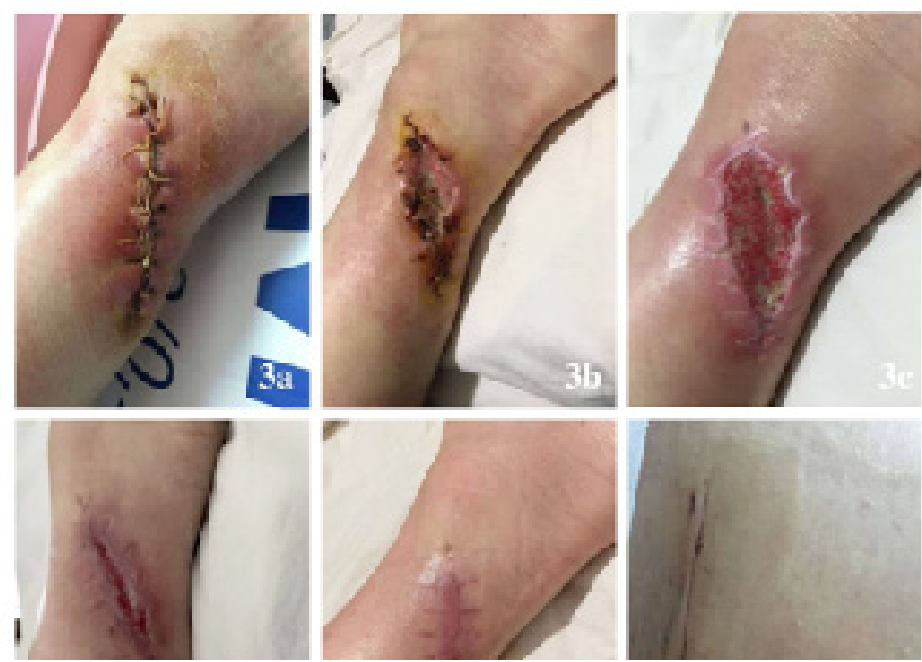

Fig 3a: Post-operative finding after secondary excision with an additional $2 \mathrm{~cm}$ surgical margin. Defect closed via single interrupted sutures. Fig 3b: Post-operative complication after second surgical session with suture disruption and wound dehiscence.

Fig 3c: Wound dehiscence complicated on an erythematous bed with oedema and complete exposure of underlying tissues.

Fig 3d: Post debridement of indurated tissues with progressive healing of the wound.

Fig 3e: Final clinical picture with complete wound closure and successful wound healing.

Fig 3f: Clinical picture after sentinel lymph node removal in the left inguinal region. Procedure parallel with secondary re-excision

Citation: Kandathil LJ, Oliveira N, Patterson JW, Tchernev G (2021). Borderline Intermediate Thickness Cutaneous Melanoma Class B: Isn't it Time for Personalised One Step Surgical Approach as Standard Clinical Behaviour?. Clin Res Dermatol Open Access 8(2): 


\section{References}

1. Swetter S, Tsao H, Bichakjian C, Lewandrowski C, Elder D, Gershenwald $\mathrm{J}$, et al. Guidelines of care for the management of primary cutaneous melanoma. J Am Acad Dermatol. 2019;80(1):208-250. https:// pubmed.ncbi.nlm.nih.gov/30392755/

2. Mays M, Martin R, Burton A, Ginter B, Edwards M, Reintgen $\mathrm{D}$, et al. Should all patients with melanoma between 1- and 2-mm Breslow thickness undergo sentinel lymph node biopsy? Cancer. 2010;116(6):1535-1544. https://pubmed.ncbi.nlm.nih. gov/20108306/

3. Fernández I, De Troya M, Fúnez R, Rivas F, Blanco G, Blázquez N. Preoperative 15-MHz ultrasound assessment of tumor thickness in malignant melanoma. Actas Dermosifiliogr. 2013;104(1):227-231. https://pubmed.ncbi.nlm.nih.gov/22938997/

4. Tchernev G. One Step Surgery for Cutaneous Melanoma: "We Cannot Solve Our Problems with the Same Thinking We Used When We Created Them?" Open Access Maced J Med Sci. 2017;5(6):774-776. https://pubmed.ncbi.nlm.nih.gov/29104688/

5. Garbe C, Amaral T, Peris K, Hauschild A, Arenberger P, Bastholt L, et al. European consensus-based interdisciplinary guideline for melanoma.
Part 2: Treatment - Update 2019. Euro J Cancer. 2020;126(1):159177. https://pubmed.ncbi.nlm.nih.gov/31866016/

6. Tchernev G, Chokoeva A. New Safety Margins for Melanoma Surgery: Nice Possibility for Drinking of "Just That Cup of Coffee"? Open Access Maced J Med Sci. 2017;5(3):352-358. https://pubmed.ncbi.nlm.nih. gov/28698757/

7. Chaput L, Laurent E, Pare A, Sallot A, Mourtada Y, Ossant F, et al. Onestep surgical removal of cutaneous melanoma with surgical margins based on preoperative ultrasound measurement of the thickness of the melanoma. Eur J Dermatol. 2018;28(1):202-208. https://pubmed. ncbi.nlm.nih.gov/29620001/

8. Russo F. One-Step surgical removal of a cutaneous melanoma: Current evidence. Actas Dermosifiliogr. 2020;111(7):541-544. https:// pubmed.ncbi.nlm.nih.gov/32553988/

9. Tchernev G, Temelkova I. The One Step Melanoma Surgery (OSMS): A New Chance for More Adequate Surgical Treatment of Melanoma Patients!? Open Access Maced J Med Sci. 2019;7(3):504-506. https:// www.ncbi.nlm.nih.gov/pmc/articles/PMC6390149/ 